\title{
Integer charge transfer at the tetrakis(dimethylamino)ethylene/Au interface
}

\author{
Lindell, L.; Unge, Mikael; Osikowicz, W.; Stafstrom, S.; Salaneck, W.R.; Crispin, X.; de Jong, M.P.
}

Published in:

Applied Physics Letters

Link to article, DOI:

$10.1063 / 1.2912818$

Publication date:

2008

Document Version

Publisher's PDF, also known as Version of record

Link back to DTU Orbit

Citation (APA):

Lindell, L., Unge, M., Osikowicz, W., Stafstrom, S., Salaneck, W. R., Crispin, X., \& de Jong, M. P. (2008). Integer charge transfer at the tetrakis(dimethylamino)ethylene/Au interface. Applied Physics Letters, 92(16), 163302. https://doi.org/10.1063/1.2912818

\section{General rights}

Copyright and moral rights for the publications made accessible in the public portal are retained by the authors and/or other copyright owners and it is a condition of accessing publications that users recognise and abide by the legal requirements associated with these rights.

- Users may download and print one copy of any publication from the public portal for the purpose of private study or research.

- You may not further distribute the material or use it for any profit-making activity or commercial gain

- You may freely distribute the URL identifying the publication in the public portal 


\title{
Integer charge transfer at the tetrakis(dimethylamino)ethylene/Au interface
}

\author{
Linda Lindell, ${ }^{1}$ Mikael Unge, ${ }^{2}$ Wojciech Osikowicz, ${ }^{1}$ Sven Stafström, ${ }^{1}$ William R. Salaneck, ${ }^{1}$ \\ Xavier Crispin, ${ }^{3, a)}$ and Michel P. de Jong ${ }^{4}$ \\ ${ }_{1}^{1}$ Department of Physics, Chemistry and Biology, Linköping University, S-581 83 Linköping, Sweden \\ ${ }^{2}$ Department of Physics, Technical University of Denmark, DK-2800 Lyngby, Denmark \\ ${ }^{3}$ Department of Science and Technology, Campus Norrköping, Linköping University, \\ S-60174 Norrköping, Sweden \\ ${ }^{4}$ MESA + Institute for Nanotechnology, University of Twente, 7500 AE Enschede, The Netherlands
}

(Received 11 December 2007; accepted 23 March 2008; published online 21 April 2008)

\begin{abstract}
In organic-based electronics, interfacial properties have a profound impact on device performance. The lineup of energy levels is usually dependent on interface dipoles, which may arise from charge transfer reactions. In many applications, metal-organic junctions are prepared under ambient conditions, where direct overlap of the organic $\pi$ system from the metal bands is prevented due to presence of oxides and/or hydrocarbons. We present direct experimental and theoretical evidence showing that the interface energetic for such systems is governed by exchange of an integer amount of electrons. (C) 2008 American Institute of Physics. [DOI: 10.1063/1.2912818]
\end{abstract}

In state of the art organic electronics, device performance and lifetime crucially depend on the properties of both the active materials and their interfaces. It is an unfortunate fact that the dramatic progress which has been recently achieved in materials design and manufacturing has not been matched by an equal improvement in interface engineering. Consequently, devices typically contain a large number of layers with different functions that could, ultimately, be replaced by several "smart" interfaces. Significant interface dipoles are routinely observed when a conjugated molecule is deposited in vacuum onto an atomically clean metal surface because of the direct mixing of its $\pi$-orbitals with the metallic bands upon covalent-type chemisorption. The origin of the interfacial dipole at the organic-metal interface has been investigated by following the change in the metal work function $\Delta \mathrm{WF}$ upon adsorption of the organic monolayer. $^{1-4}$

Here, we focus on a specific class of interfaces that is very common in a large range of applications, namely, weakly interacting systems of $\pi$-conjugated molecules (or polymers) on metallic contacts. Indeed, for ex situ prepared interfaces by solution processing, the direct overlap between the $\pi$-orbitals of the conjugated materials (polymer or molecule) and the metal surface is often prevented by either surface oxide and/or hydrocarbon surface contaminations. However, even for such cases significant dipoles have been observed. $^{5-7}$ Upon varying the work function of the substrate, abrupt transitions are observed between a SchottkyMott regime, in which the interface dipole is negligible, and Fermi-level pinning regimes, where the size of the dipole scales with the difference between the adiabatic (i.e., fully relaxed, electronically, and geometrically) ionization potential or electron affinity of the conjugated polymer and the work function of the substrate. ${ }^{6}$ This is explained by spontaneous charge transfer between the electrode and the conjugated polymer via tunneling (integer charge transfer), but it has been never directly observed.

In this letter, we report a joint experimental and theoretical study of the tetrakis(dimethylamino)ethylene

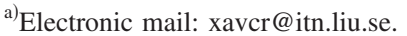

(TDAE)/Au interface by photoelectron spectroscopy and quantum chemical modeling. TDAE is used as a model system that mimics the situation of an organic semiconductor, with an electron donor character, that has no direct overlap between its $\pi$-molecular orbitals and the metal bands. The TDAE molecule has a strong electron donor character due to the presence of four amine groups providing an excess of electron density on the central vinylic group $(\mathrm{C}=\mathrm{C}$ bond $)$. The electron rich $\mathrm{C}=\mathrm{C}$ bond is encased by eight outer methyl groups which prevents the overlap between molecular orbitals and metal bands.

Polycrystalline gold substrates were sputter cleaned in ultrahigh vacuum (UHV) and thereafter cooled down before exposure to the vapor of TDAE. At a temperature just above the condensation temperature of TDAE (about $200 \mathrm{~K}$ in UHV) a stable submonolayer of TDAE is formed. ${ }^{8}$ In the following, we will simply refer to this stable coverage as a monolayer, although it should be noted that the areal density is considerably less than that of a densely packed single layer of TDAE molecules. The work function of the bare gold surface is $5.2 \mathrm{eV}$. Upon exposure to TDAE, UV photoelectron spectroscopy (UPS) reveals that the work function of the TDAE-modified gold surface decreases by 1.3 down to $3.9 \mathrm{eV}$. For the sake of comparison, the change in work function for a polycrystalline gold surface due to hydrocarbon contamination arising from air exposure is about $0.7 \mathrm{eV}$ and attributed mostly to the Pauli repulsion. The magnitude of the shift resulting from TDAE absorbed on the gold thus shows that additional effects are involved. Since the molecule itself does not carry an intrinsic dipole in its neutral or charged state, ${ }^{9}$ the large work function change can only be explained by a charge transfer reaction between TDAE and $\mathrm{Au}$.

In Fig. 1, the UPS ( $h \nu=21.2 \mathrm{eV})$ valence band spectra of gold (dotted line), a TDAE monolayer on gold (full line), and a TDAE multilayer (dashed line) are shown. In addition to these spectra, Fig. 1 also includes simulated spectra of different charge states of TDAE as well as a gas-phase UPS spectrum. The theoretical simulations (adapted from Osikowicz et al. ${ }^{10}$ ) are based on Koopmans' approximation; i.e., using density functional theory eigenvalues (B3LYP hy- 


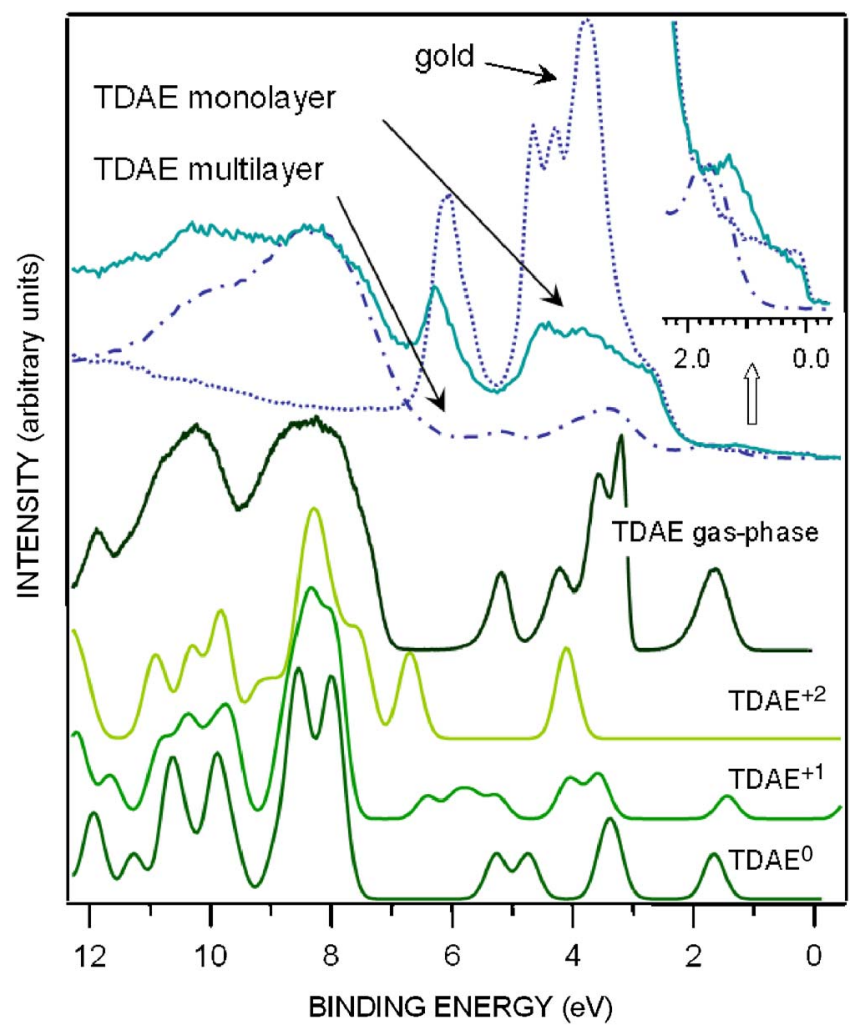

FIG. 1. (Color online) The UPS spectra of the clean gold surface (dotted line), the TDAE monolayer on gold (full line), and a multilayer of TDAE (dashed-dotted line), together with an enlargement of the lowest part of the valence band, are displayed in the upper part of the figure. Below, the calculated spectra of the different charged states are plotted together with a gas-phase measurement of TDAE. The binding energies are vs the gold Fermi level.

brid exchange-correlation functional) as binding energies, without estimating cross sections, and by matching the highest occupied molecular orbital (HOMO) of the single molecule to the ionization potential in the UPS spectrum (versus vacuum level) obtained for the TDAE multilayer in order to account for solid-state polarization effects. For more details, see Ref. 10.

The presence of TDAE on the Au surface is clearly revealed by the appearance of the two peaks located between 8 and $10 \mathrm{eV}$ attributed to the $\sigma$-levels of the molecule (Fig. 1). Those levels are used as an internal reference when comparing the monolayer and multilayer signals. As can be seen from the calculated UPS spectra, the $\mathrm{TDAE}^{+}$radical cation is characterized by the destabilization of the HOMO. The destabilization of the HOMO is due to elongation of the central $\mathrm{C}=\mathrm{C}$ bond upon oxidation resulting from a reduction of the bonding character of the $\pi$-bond. Such an effect is similar to the formation of polaron levels in the band gap of extended $\pi$-systems, such as conjugated polymers. The dication $\mathrm{TDAE}^{2+}$, obtained after removal of two electrons from the HOMO level of TDAE, does not contribute to the photoelectron signal intensity in this energy region. Hence, the modification of the HOMO of TDAE upon adsorption on gold can reveal the charge state of the molecule.

In Fig. 1, all spectra except those of Au and the TDAE monolayer on $\mathrm{Au}$, are shifted to align the peaks originating from the $\sigma$-orbtials of TDAE, to that found for the chemisorbed TDAE monolayer used as an internal reference level as mentioned above. The comparison between the spectra of the multilayer and that of the TDAE monolayer reveals a

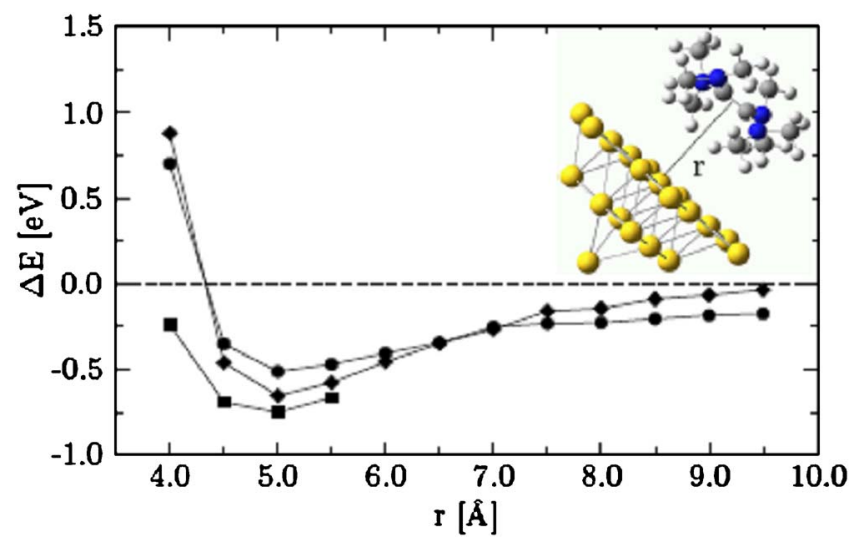

FIG. 2. (Color online) Chemisorption energy as a function of $r$ for the $\mathrm{Au}_{20}$-TDAE complex. Different geometries of the molecule have been used. Neutral molecule (circles), singly charged molecules (diamonds), and relaxed molecular geometry (squares). The inset shows the geometry of the complex.

clear destabilization of the low binding energy peak associated with the HOMO from 1.8 to $1.2 \mathrm{eV}$. This $0.6 \mathrm{eV}$ shift toward high binding energy indicates the presence of singly charged molecular radical cation $\mathrm{TDAE}^{+1}$ on gold in agreement with the theoretical simulations. This not only proves that positively charged species are formed, but also indicates that the amount of charge transfer must be nearly integer. In contrast, partial charge transfer accompanied by hybridization with the Au bands would lead to a wide distribution of states, ${ }^{4}$ and not a resolvable singly occupied molecular orbital (SOMO).

In addition to the experimental results, first principles calculations are performed on models for the TDAE/Au interface. The gold (111) surface is modeled in the calculations by a $\mathrm{Au}_{20}$ cluster with a top layer with 13 atoms, the minimal number of atoms required to fit the entire molecule on top of the surface. A second layer, which has been shown to be important for the electronic structure of thiolates on gold, ${ }^{11}$ contains six atoms and a third layer, a single atom, introduced to get an even number of gold atoms in total which yields a closed shell system. All $\mathrm{Au}-\mathrm{Au}$ bond distances are set to $2.88 \AA$. TDAE is positioned in such a way that the axis through the $\mathrm{C}=\mathrm{C}$ bond is parallel to the surface and the midpoint of the $\mathrm{C}=\mathrm{C}$ bond is above the center gold atom in the cluster. This distance is called $r$ in the inset of Fig. 2. The calculations were carried out using the GAUSSIAN 03 (Ref. 12) program, with the B3LYP hybrid exchangecorrelation functional. ${ }^{13,14}$ Hence, in the calculation of TDAE on gold, we used the 6-31G and cc-pVDZ basis sets for the light elements $(\mathrm{H}, \mathrm{C}$, and $\mathrm{N})$ and the relativistic corrected basis set LanL2DZ with effective core potentials for gold. ${ }^{15}$

The geometrical structure of the isolated neutral TDAE molecule and radical cation $\mathrm{TDAE}^{+}$were fully optimized (without symmetry constraints). In agreement with experimental x-ray diffraction data for the $\mathrm{TDAE}^{+} \mathrm{C}_{60}^{-}$materials, ${ }^{9}$ the oxidation of TDAE leads to an elongation of $\mathrm{C}=\mathrm{C}$ bond in the central vinylic group and a decrease of the $\mathrm{C}-\mathrm{N}$ bond length as expected from the removal of an electron from the HOMO that has a bonding character on the $\mathrm{C}=\mathrm{C}$ bond and antibonding character on the $\mathrm{C}-\mathrm{N}$ bond.

Since the TDAE molecule interacts with the model gold surface with mostly nondirectional bonds (see below), the chemisorption energy is less sensitive to the exact position of AIP license or copyright; see http://apl.aip.org/apl/copyright.jsp 
the molecule. ${ }^{1}$ We therefore fix the position of the midpoint of the $\mathrm{C}=\mathrm{C}$ bond to that above the center gold atom in all calculations. The chemisorption energy $\Delta E$ is defined as the difference between the total energy $E$ of the TDAE/ $\mathrm{Au}_{20}$ complex, $E^{\mathrm{TDAE} / \mathrm{Au}_{20}}$, and the sum of the total energies of the remote parts (TDAE and $\mathrm{Au}_{20}$ )

$$
\Delta E=E^{\mathrm{TDAE} / \mathrm{Au}_{20}}-\left(E^{\mathrm{TDAE}}+E^{\mathrm{Au}}\right) .
$$

Hence, negative chemisorption energy means that the adsorption is stable. As a first approach, the potential energy curves for the TDAE-Au interaction, shown in Fig. 2, are directly estimated from the chemisorption energy calculated for different distances $r$ using gas-phase geometries of neutral (circles), singly (diamonds) and doubly charged molecules in the TDAE/ $/ \mathrm{Au}_{20}$ complex. For each distance $r$, the electron density of the complex is optimized via the selfconsistent field procedure. A minimum of the chemisorption energy is found for the neutral and singly charged molecule at about $r=5.0 \AA$, while the calculation yields positive values of chemisorption energy using the geometry of the doubly charged molecule. In agreement with experimental observations, two-electron transfer from TDAE to the gold surface is not taking place since the adsorption of $\mathrm{TDAE}^{2+}$ is not stable. To further improve the accuracy of the result we let the internal structure of the singly charged molecule relax on the surface when calculating $E^{\mathrm{TDAE} / \mathrm{Au}_{20}}$ (squares in Fig. 2). To reduce the computational time, these calculations are only performed for a few values of $r$ around the energy minimum obtained above. The chemisorption is further stabilized $(\Delta E=-0.73 \mathrm{eV})$ compared to the unrelaxed system $(\Delta E$ $=-0.66 \mathrm{eV}$ ) but the position of the energy minimum is unchanged. Note that for $r$ larger than $7.0 \AA$ the complex with the neutral gas-phase geometry has higher chemisorption energy than the complex for which the geometry of the singly charged molecule is used. This is expected since the charge transfer from the molecule to the cluster decreases when $r$ is increased, and the molecule becomes more stable for the neutral structure above a certain value of $r$.

The analysis of the charge distribution, following Mulliken's scheme, for the relaxed system shows an almost complete charge transfer from the TDAE to the gold cluster $\left(\Delta q_{\mathrm{TDAE}} / e=0.91\right.$ for the relaxed system, $\Delta q_{\mathrm{TDAE}} / e=0.93$ for the unrelaxed system), in agreement with the experimental observations. Most of the excess positive charge (72\%) on TDAE is located on the carbon atoms of the central vinylic group. In the TDAE/ $\mathrm{Au}_{20}$ complex, this orbital is partially depopulated as a result of the charge transfer with the gold surface. The bond length of the $\mathrm{C}=\mathrm{C}(\mathrm{N}-\mathrm{C})$ bond is $1.41 \AA$ $(1.39 \AA)$ in the TDAE/ $\mathrm{Au}_{20}$ complex. Those bond lengths are similar to those obtained for an isolated singly charged TDAE. However, the dihedral angle around the central $\mathrm{C}=\mathrm{C}$ bond in the chemisorbed TDAE is $28^{\circ}$, which is considerably smaller than for the singly charged molecule in the gas phase. This is attributed to the electrostatic contribution in the chemisorption energy that forces the cationic $\mathrm{TDAE}^{+}$ to come close to the gold surface and partially overcomes the rotation barrier around the central $\mathrm{C}=\mathrm{C}$ bond.

In summary, we present experimental and theoretical evidence that the interface energetics for weakly interacting systems of $\pi$-conjugated molecules (or polymers) on metallic contacts are governed by the exchange of an integer amount of electrons, as opposed to partial charge transfer in hybridized systems.

UPS measurements reveal a destabilization of the SOMO level of the adsorbate by around $0.6 \mathrm{eV}$ versus the HOMO of the neutral molecule and a significant work function decrease of about $1.3 \mathrm{eV}$ upon monolayer adsorption resulting from the strong electric dipoles formed upon electron transfer from TDAE to Au. Robust quantum chemical models indicate that TDAE is strongly chemisorbed on the model gold surface and carries a unitary positive charge. The negative charge at the metal surface and the positive charge carried by the molecular adsorbate protected with its methyl groups make the electrostatic contribution important to the chemisorption energy. This type of interaction can be described as a molecular ionic chemisorption.

Research in Norrköping and Linköping is supported by the Swedish Foundation for Strategic Research (SSF) through the project COE, the EU Integrated Project NAIMO (No. NMP4-CT-2004-500355) and by the Swedish Science Research Council (VR).

${ }^{1}$ X. Crispin, V. Geskin, A. Crispin, J. Cornil, R. Lazzaroni, W. R. Salaneck, and J. L. Bredas, J. Am. Chem. Soc. 124, 8131 (2002).

${ }^{2}$ H. Ishii, K. Sugiyama, E. Ito, and K. Seki, Adv. Mater. (Weinheim, Ger.) 11, 605 (1999).

${ }^{3}$ N. Koch, S. Duhm, J. P. Rabe, A. Vollmer, and R. L. Johnson, Phys. Rev. Lett. 95, 237601 (2005)

${ }^{4}$ L. Lindell, M. P. de Jong, W. Osikowicz, R. Lazzaroni, M. Berggren, W. R. Salaneck, and X. Crispin, J. Chem. Phys. 122, 084712 (2005).

${ }^{5}$ C. Tengstedt, W. Osikowicz, W. R. Salaneck, I. D. Parker, C.-H. Hsu, and M. Fahlman, Appl. Phys. Lett. 88, 053502 (2006).

${ }^{6}$ A. Crispin, X. Crispin, M. Fahlman, M. Berggren, and W. R. Salaneck, Appl. Phys. Lett. 89, 213503 (2006).

${ }^{7}$ S. Braun, W. Osikowicz, Y. Wang, and W. R. Salaneck, Org. Electron. 8, 14 (2007).

${ }^{8}$ L. Lindell, A. Burquel, F. L. E. Jakobsson, V. Lemaur, M. Berggren, R. Lazzaroni, J. Cornil, W. R. Salaneck, and X. Crispin, Chem. Mater. 18, 4246 (2006).

${ }^{9}$ K. I. Pokhodnia, J. Papavassiliou, P. Umek, A. Omerzu, and D. Mihailovic, J. Chem. Phys. 110, 3606 (1999).

${ }^{10}$ W. Osikowicz, X. Crispin, C. Tengstedt, L. Lindell, T. Kugler, and W. R. Salaneck, Appl. Phys. Lett. 85, 1616 (2004).

${ }^{11}$ H. Sellers, A. Ulman, Y. Shnidman, and J. E. Eilers, J. Am. Chem. Soc. 115, 9389 (1993).

${ }^{12}$ M. J. Frisch, G. W. Trucks, H. B. Schlegel, G. E. Scuseria, M. A. Robb, J. R. Cheeseman, J. A. Montgomery, Jr., T. Vreven, K. N. Kudin, J. C. Burant, J. M. Millam, S. S. Iyengar, J. Tomasi, V. Barone, B. Mennucci, M. Cossi, G. Scalmani, N. Rega, G. A. Petersson, H. Nakatsuji, M. Hada, M. Ehara, K. Toyota, R. Fukuda, J. Hasegawa, M. Ishida, T. Nakajima, Y. Honda, O. Kitao, H. Nakai, M. Klene, X. Li, J. E. Knox, H. P. Hratchian, J. B. Cross, V. Bakken, C. Adamo, J. Jaramillo, R. Gomperts, R. E. Stratmann, O. Yazyev, A. J. Austin, R. Cammi, C. Pomelli, J. W. Ochterski, P. Y. Ayala, K. Morokuma, G. A. Voth, P. Salvador, J. J. Dannenberg, V. G. Zakrzewski, S. Dapprich, A. D. Daniels, M. C. Strain, O. Farkas, D. K. Malick, A. D. Rabuck, K. Raghavachari, J. B. Foresman, J. V. Ortiz, Q. Cui, A. G. Baboul, S. Clifford, J. Cioslowski, B. B. Stefanov, G. Liu, A. Liashenko, P. Piskorz, I. Komaromi, R. L. Martin, D. J. Fox, T. Keith, M. A. Al-Laham, C. Y. Peng, A. Nanayakkara, M. Challacombe, P. M. W. Gill, B. Johnson, W. Chen, M. W. Wong, C. Gonzalez, and J. A. Pople, Gaussian, Inc., Wallingford CT, 2004.

${ }^{13}$ A. D. Becke, J. Chem. Phys. 98, 5648 (1993).

${ }^{14}$ C. Lee, W. Yang, and R. G. Parr, Phys. Rev. B 37, 785 (1988).

${ }^{15}$ P. J. Hay and W. R. Wadt, J. Chem. Phys. 82, 299 (1985). 